\title{
Effects of a metabolic optimized fast track concept (MOFA) on bowel function and recovery after surgery in patients undergoing elective colon or liver resection: a randomized controlled trial
}

\author{
Christopher Uhlig $^{1 *}\left(\mathbb{D}\right.$, Thomas Rössel ${ }^{1}$, Axel Denz ${ }^{2,3}$, Sven Seifert ${ }^{2,4}$, Thea Koch ${ }^{1}$ and Axel Rüdiger Heller ${ }^{1,5}$
}

\begin{abstract}
Background: Enhanced recovery after surgery programs (ERAS) using thoracic epidural anesthesia and perioperative patient conditioning with omega-3 fatty acids (n3FA), glucose control (GC) and on-demand fluid therapy, respectively, showed beneficial effects. In the MOFA- study these components were used together in patients undergoing colon or liver surgery. We hypothesized that the use of a perioperative MOFA program improves intestine function represented as time to the first postoperative bowel movement in adult patients compared to standard ERAS.
\end{abstract}

Methods: After BfArM and IRB approval 100 patients were enrolled in this prospective randomized controlled trial. All patients received ERAS therapy (control). In addition, the MOFA group received $0.2 \mathrm{~g} / \mathrm{kg}$ fish oil (Omegaven ${ }^{\oplus}$ ), preoperatively, followed by a $48 \mathrm{~h}$ continuous infusion of $0.2 \mathrm{~g} / \mathrm{kg} / \mathrm{d}$ n3FA; and GC was kept below < $8 \mathrm{mmol} /$ L. Pre- and postoperatively energy drinks were administered.

Results: As compared to control group the MOFA concept resulted in an earlier onset of flatulence by $14 \mathrm{~h}$ (46.6 $\pm 25.7,32.0 \pm 17.9, p=0.030$, hours, control vs. MOFA, respectively). Effects on onset of bowel movement were not observed $(74.5 \pm 30.4,66.4 \pm 29.2, p=0.163$, hours, control vs. MOFA, respectively). The disease severity (SAPS II score; $p=0.720$ ) as well as deployment of resources (TISS 28 score, $p=0.709$ ) did not differ between groups. No statistic significant difference between MOFA and control group regarding inflammation, impairment of coagulation, length of hospital stay or incidence of postoperative surgical complications were observed.

Conclusions: The MOFA concept did not result in an improvement of intestine function or faster recovery after elective colon or liver surgery compared to standard ERAS therapy. Omega-3 fatty acids showed no impairment of coagulation or improved resolution of inflammation. Further trials in a larger patient collective are needed to investigate potential beneficial effects of omega-3 fatty acids in abdominal surgery.

Trial registration: This trial was prospectively registered at the European Union Clinical Trials Register (EuDraCT 2005-004814-33, date: 10-05-2005, https://www.clinicaltrialsregister.eu/ctr-search/search?query=2005004814-33+).

Keywords: Omegaven, Abdominal surgery, Liver surgery, Colon surgery, Omega-3 fatty acids, ERAS, Recovery after surgery, Randomized controlled trial

\footnotetext{
* Correspondence: christopher.uhlig@ukdd.de

'Department of Anaesthesiology and Critical Care Medicine, University

Hospital Carl Gustav Carus at the Technische Universität Dresden, Fetscherstr.

74, 01307 Dresden, Germany

Full list of author information is available at the end of the article
}

(c) The Author(s). 2019 Open Access This article is distributed under the terms of the Creative Commons Attribution 4.0 International License (http://creativecommons.org/licenses/by/4.0/), which permits unrestricted use, distribution, and reproduction in any medium, provided you give appropriate credit to the original author(s) and the source, provide a link to the Creative Commons license, and indicate if changes were made. The Creative Commons Public Domain Dedication waiver (http://creativecommons.org/publicdomain/zero/1.0/) applies to the data made available in this article, unless otherwise stated. 


\section{Background}

Within the past decade enhanced recovery after surgery (ERAS) programs were established covering a multitude of surgical interventions. Besides others, a meta-analysis on ERAS in different type of hepatectomy clearly showed improved patient outcome [1]. Aside minimizing surgical stress and improving pain control one key success factor for further developments of ERAS programs is the increasing control of noxious circumstances such as overwhelming metabolism [2].

Overshooting early up-regulation of host defense after complex surgery induces severe tissue injury, potentially culminating systemic inflammatory response with unfavorable outcome [3, 4]. Omega-3 fatty acids (n3FA) are capable of dampening early hyperinflammatory processes, by changing cell to cell signal transduction as shown [5]. Later features of host defense, however, are enhanced by n3FA without inducing hyperinflammatory states.

The n3FA have shown their efficacy in the treatment of chronic and acute inflammatory diseases [3] due to their pleiotropic effects on inflammatory cell signalling pathways [6-8]. In a variety of experimental $[9,10]$ and clinical studies omega-3 fatty acids attenuated hyperinflammatory conditions and induced faster recovery [11]. By reducing length of antibiotic therapy and hospital stay [12], even in critically ill patients, net cost savings are possible from the use of n3FA despite higher expenditure for the prescriptions [13].

In a prospective, open label, multicenter case series in 661 patients receiving parenteral fish oil our group evaluated survival, length of intensive care unit (ICU) stay, hospital stay and use of antibiotics, with respect to the primary diagnosis and extent of organ dysfunction [12]. Compared to the subgroup receiving less than $0.05 \mathrm{~g} / \mathrm{kg} /$ $\mathrm{d}$ of fish oil, significantly more patients survived when $0.1-0.2 \mathrm{~g} / \mathrm{kg} / \mathrm{d}$ were administered.

Based on this data, the present study was designed to evaluate the effectiveness of an ERAS program supplemented with a priming dose of fish oil in the preoperative evening and a $48 \mathrm{~h}$ continuous infusion of $0.2 \mathrm{~g} / \mathrm{kg} / \mathrm{d}$ (so called 'Metabolic Optimized FAst track concept' [MOFA]) starting at the time of inducing anesthesia in patients undergoing elective hepatic and colon surgery.

We hypothesized that the use of a perioperative MOFA program improves intestine function represented as time to the first postoperative bowel movement in adult undergoing elective colon or hepatic surgery compared to controls without MOFA. Second, we hypothesized that the use of a perioperative MOFA program results in faster postoperative recovery represented by lower Simplified Acute Physiology Score (SAPS II) [14] as well as Simplified Therapeutic Scoring System values (TISS 28) [15] in adult patients undergoing elective colon or hepatic surgery compared to controls without MOFA.

\section{Methods}

The present prospective, single blind, mono-center, randomized controlled trial was approved by the competent authorities, Bundesinstitut für Arzneimittelsicherheit und Medizinprodukte (BfArM, GZ 61-3910-4,031,476), and Institutional Review Board of the Technische Universität Dresden (AZ 193112005), as well as registered at European Union Drug Review Agency Clinical Trials (EuDraCT 2005-004814-33). The manuscript is reported according to the CONSORT guidelines [16].

\section{Patients and randomization}

Adult patients $(18<$ age $>85$ years $)$ undergoing elective colon (tumor or diverticulosis surgery) or liver surgery (liver tumors independent from dignity without biliodigestive anastomosis) in the Universtiy Hospital Carl Gustav Carus (at the Technische Universität Dresden, Dresden, Germany) were eligible for the trial. Exclusion criteria were summarized in Additional file 1: Table S1. Convenience sampling was used as recruiting strategy and subjects were included over a three year period. Patients were randomized in blocks of four generated by a computer program either to standard ERAS (control) or MOFA group. To achieve homogeneity between groups an a priori stratification of the randomization was performed according to three strata: surgical procedure (colon or liver), body mass index (BMI, $<30$ or $\geq 30$ ) and perioperative risk classified as low or high, if one of the following factors was present: a previous abdominal operation, American Society of Anesthesiology Physical Status 3 or higher, central liver surgery, liver tumor $>5$ $\mathrm{cm}$. Group allocation was concealed in sequentially numbered opaque closed envelopes.

\section{Experimental protocol}

Detailed interventions are described in Additional file 1: Table S2. A time course of intervention is depicted in Fig. 1.

\section{Preoperative care, anesthetic regimen and surgery}

After enrollment in the trial baseline values were taken and patients were randomized to control or MOFA group. All patients received a standard ERAS protocol with enhanced physiotherapy, short or no postoperative ICU stay and planned discharge on the forth postoperative day. In addition, the MOFA group received a priming dose of $100 \mathrm{ml}$ Omegaven $^{\circ} 10 \%$ (Fresenius Kabi AG, Bad Homburg, Germany) on the evening before surgery. Two hours before the induction of anesthesia continuous infusion of Omegaven ${ }^{\circ} 10 \%$ with $0.083 \mathrm{ml} / \mathrm{kg} / \mathrm{h}(0.2$ $\mathrm{g} / \mathrm{kg} / \mathrm{d}$ ) for $48 \mathrm{~h}$ was started and a high caloric energy drink (ProvideXtra ${ }^{\circ}$ Drink $200 \mathrm{ml}$, Fresenius Kabi AG, Bad Homburg, Germany) administered orally in the 


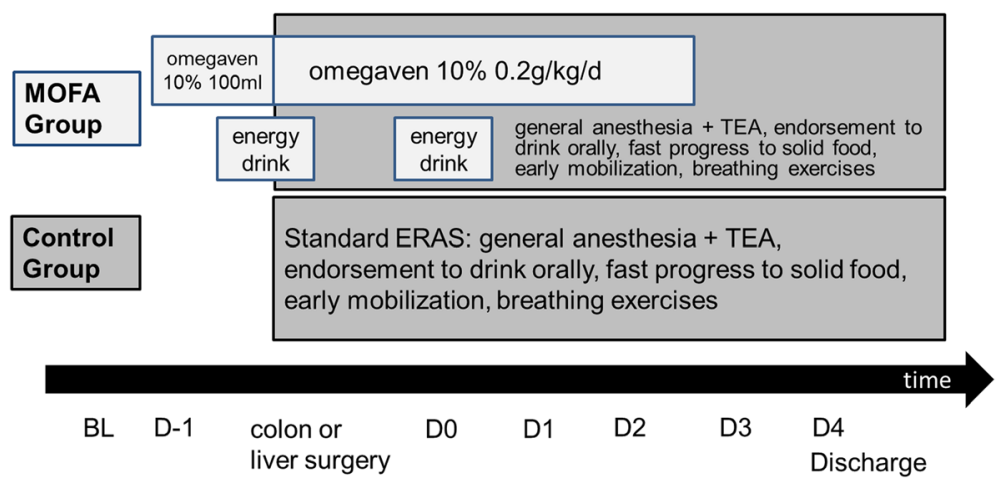

Fig. 1 Time course of interventions After enrollment in the trial baseline values were taken and patients were randomized to control or MOFA group. All patients received a standard ERAS protocol with enhanced physiotherapy, short or no postoperative intensive care unit stay and planned discharge on the forth postoperative day. In addition, the MOFA group received a priming dose of $100 \mathrm{ml}$ omegaven $10 \%$ on the evening before surgery. Two hours before the induction of anesthesia continuous infusion of omegaven $10 \%$ with $0.083 \mathrm{ml} / \mathrm{kg} / \mathrm{h}(0.2 \mathrm{~g} / \mathrm{kg} / \mathrm{d})$ for $48 \mathrm{~h}$ was started and a high caloric energy drink administered orally in the MOFA group. ERAS: enhanced recovery after surgery concept, MOFA: metabolic optimized fast track concept, TEA: thoracic epidural analgesia, BL: baseline, D-1: preoperative day, D0: day of surgery, D1-D4: postoperative day 1-4, respectively

MOFA group. All patients received general anesthesia with propofol/sufentanil for induction and volatile anesthetics in combination with sufentanil and thoracic epidural anesthesia (TEA) for maintenance. The choice of the volatile anesthetic and the neuromuscular blocking agent for continuous muscle relaxation was on the discretion of the anesthesiologist. Co-priming with $500 \mathrm{ml}$ colloid solution before the first injection of local anesthetic for the epidural anesthesia was performed in the MOFA group. An arterial line as well as a central venous catheter were placed in all patients. Intraoperative fluid was restricted to $4 \mathrm{ml} / \mathrm{kg} / \mathrm{h}$. Surgery was carried out by a predefined experienced team of specialists in abdominal surgery. All patients who underwent liver surgery received a transverse upper abdomen laparotomy and in case of colon surgery a median laparotomy or laparoscopic approach as appropriate. Patients were extubated directly after the end of surgery. Intraoperative blood glucose level was kept below $8 \mathrm{mmol} / \mathrm{L}$.

\section{Postoperative management}

Patients who received colon surgery and basic liver surgery were transferred to the post anesthesia care unit and then to normal ward. Subjects undergoing more complex liver surgery were allocated to the ICU directly after end of anesthesia. On the day of surgery oral fluids were administered postoperatively from $2 \mathrm{~h}$ on. In the MOFA group the high caloric energy drink was given and Omegaven $10 \%$ infusion was stopped at 2 nd postoperative day. Patients were endorsed to drink $>1500 \mathrm{ml}$ per day. A fast progress to solid food was intended. Physical mobilization and breathing exercises started $5 \mathrm{~h}$ after the end of surgery and were continued until discharge. Infusion was limited to $500 \mathrm{ml}$ per day. TEA was continued as patient controlled analgesia until the 2nd postoperative day and removed together with the central venous line. Blood sugar level was aimed to be kept between $4 \mathrm{mmol} / \mathrm{L}$ and $8 \mathrm{mmol} / \mathrm{L}$ in the MOFA group and between $4 \mathrm{mmol} / \mathrm{L}$ and $10 \mathrm{mmol} / \mathrm{L}$ in the control group during the first three postoperative days (Additional file 1: Table S3). Discharge was aimed from the fourth postoperative day on.

\section{Safety thresholds}

Participation in the trial was terminated at any time point, if one of the following dropout criteria was present: request of the patient, therapy refractory bowel paralysis, allergic reaction to study medication, onset of contraindications against enteral nutrition, development of septic shock and violation of safety parameters. Safety parameters to avoid bleeding complications were measured daily during the first three postoperative days. The following safety thresholds were defined: hemoglobin $>4.5 \mathrm{mmol} / \mathrm{L}$, platelets $>50 \mathrm{Gpt} /$ $\mathrm{L}$, prothrombin time $(\mathrm{PT})>50 \%$, activated partial thromboplastin time $(\mathrm{aPTT})<60$ s. If a safety threshold was violated, Omegaven infusion was stopped and reasons for violation were investigated.

\section{Measurements and data collection}

Daily visits throughout the study period were performed. Investigators collecting data were not blinded to groups. Patients were unaware of group allocation but blinding could be easily broken. Preoperative baseline data included physical examination, basic monitoring (blood pressure, peripheral oxygen saturation) and laboratory parameters (complete blood count, aPPT, PT, antithrombin, fibrinogen, blood urea nitrogen, creatinine, c-reactive protein (CRP), procalcitonin (PCT), tumor necrosis factor-alpha (TNF- $\alpha$ ), interleukin 6 (IL-6) and 10 (IL-10), blood sugar, lactate). These measurements were repeated 
until the third postoperative day. Data from the medical record were extracted daily. The presence of flatulence and/or bowel movement was documented during the first postoperative days. The TISS 28 and SAPS II scores were calculated daily.

\section{Statistics}

All calculations were performed using SPSS (IBM SPSS Statistics Vers. 23.0.0.0, IBM Deutschland GmbH, Ehningen, Germany). Graphs were computed with GraphPad Prism (Vers. 6.01, GraphPad Software Inc., La Jolla, CA, USA). Sample size was calculated based on an internal database consisting of 291 patients undergoing colon surgery, where the time to the first bowel movement was $3.25 \pm 1.7$ days. With an assumption of a two-sided $\alpha$ of 0.05 and a statistical power of $0.80,47$ patients per group were calculated to be necessary to accelerate the onset of bowel movement (primary endpoint) by one day. To compensate for dropouts, a sample size of 50 per group was projected. Distribution of the data was assessed visually using Q-Q-Plot. Patient data were analyzed as intention to treat. No assumption for missing data was done. Values are given as median, $25 \%$ percentile, $75 \%$ percentile, minimum and maximum; mean \pm standard deviation or absolute number (percentage); as appropriate. The analysis of the primary endpoint was performed with log rank test and cox regression. A two-sided Student's t-test or Mann-Whitney U test were used as appropriate for binary outcomes. Statistical significance between groups for repeated measures as well as time effect and time vs. group effect were calculated with a general linear model using the respective baseline data as covariate. Statistical significance was considered to be at two-sided $p<0.05$.

\section{Results}

The consort diagram is depicted in Fig. 2. Out of 347 screened patients, 100 consecutive patients were enrolled in the trial. Various dropouts, mainly caused by more complex resections than planned due to intraoperative tumor spread, resulted in 79 patients $(n=37$ control, $n=$ 42 MOFA group) with available follow up data. Baseline characteristics are summarized in Table 1.

\section{Bowel function}

Postoperative bowel function is shown in Table 2. With the MOFA concept an earlier onset of discharge of gas by $14 \mathrm{~h}(46.6 \pm 25.7,32.0 \pm 17.9, p=0.030$, hours, control vs. MOFA, respectively) was detected. However, there was no statistic significant difference in bowel movement $(74.5 \pm 30.4,66.4 \pm 29.2, p=0.163$, hours, control vs. MOFA, respectively) or the administration of agents for bowel stimulation.

\section{Recovery after surgery}

As shown in Fig. 3, no statistic significant difference in disease severity, represented by the SAPS II score was observed between control and MOFA group $(p=0.720)$. The deployment of resources measured by the TISS 28 score did not differ between groups (Fig. 3). Duration of anesthesia and surgery, intraoperative blood loss, overall amount of administered crystalloids and transfusions were comparable between groups (Table 3). Patients undergoing liver surgery received more crystalloids in the control group compared to MOFA group. Postoperative surgical complications were higher in the control group causing trial dropouts mainly due to insufficiency of anastomosis with consecutive reoperation and impossible enteral nutrition (Table 3). There were no statistic significant differences, neither in length of ICU nor in length of hospital stay (Table 3). There was one in-hospital death in the control group due to myocardial infarction with consecutive multi-organ failure after cardiac arrest.

\section{Hemodynamics, clinical chemistry and metabolic data} Hemodynamics are summarized in Additional file 1: Table S4. No difference between groups were detected. The blood sugar level was kept below 10 $\mathrm{mmol} / \mathrm{L}$ in the control group and below $8 \mathrm{mmol} / \mathrm{L}$ in the MOFA group (Additional file 1: Figure S1) resulting in a higher insulin administration in the MOFA group (Additional file 1: Table S5). There was no difference in serum values of parameters of liver injury and function (Additional file 1: Figure S3). Both groups had comparable kidney function (Additional file 1: Table S6).

\section{Inflammation}

Pro- and anti-inflammatory cytokines as well as clinical relevant parameters of inflammation are depicted in Additional file 1: Figure S4. No difference between groups in CRP, PCT, IL-6, TNF- $\alpha$ or IL-10 were observed in the MOFA group compared to control.

\section{Safety thresholds and investigational drug related adverse events}

Coagulation parameters were comparable between groups (Additional file 1: Figure S2) and intraoperative blood loss was comparable. Severe postoperative bleeding complications did not occur in the MOFA group compared to control measured on need for transfusion (Table 3) and hemoglobin and hematocrit levels were comparable between groups (Additional file 1: Table S6). However, in one patient, who underwent liver surgery with unplanned high intraoperative blood loss, Omegaven ${ }^{\bullet}$ was stopped precautiously on 


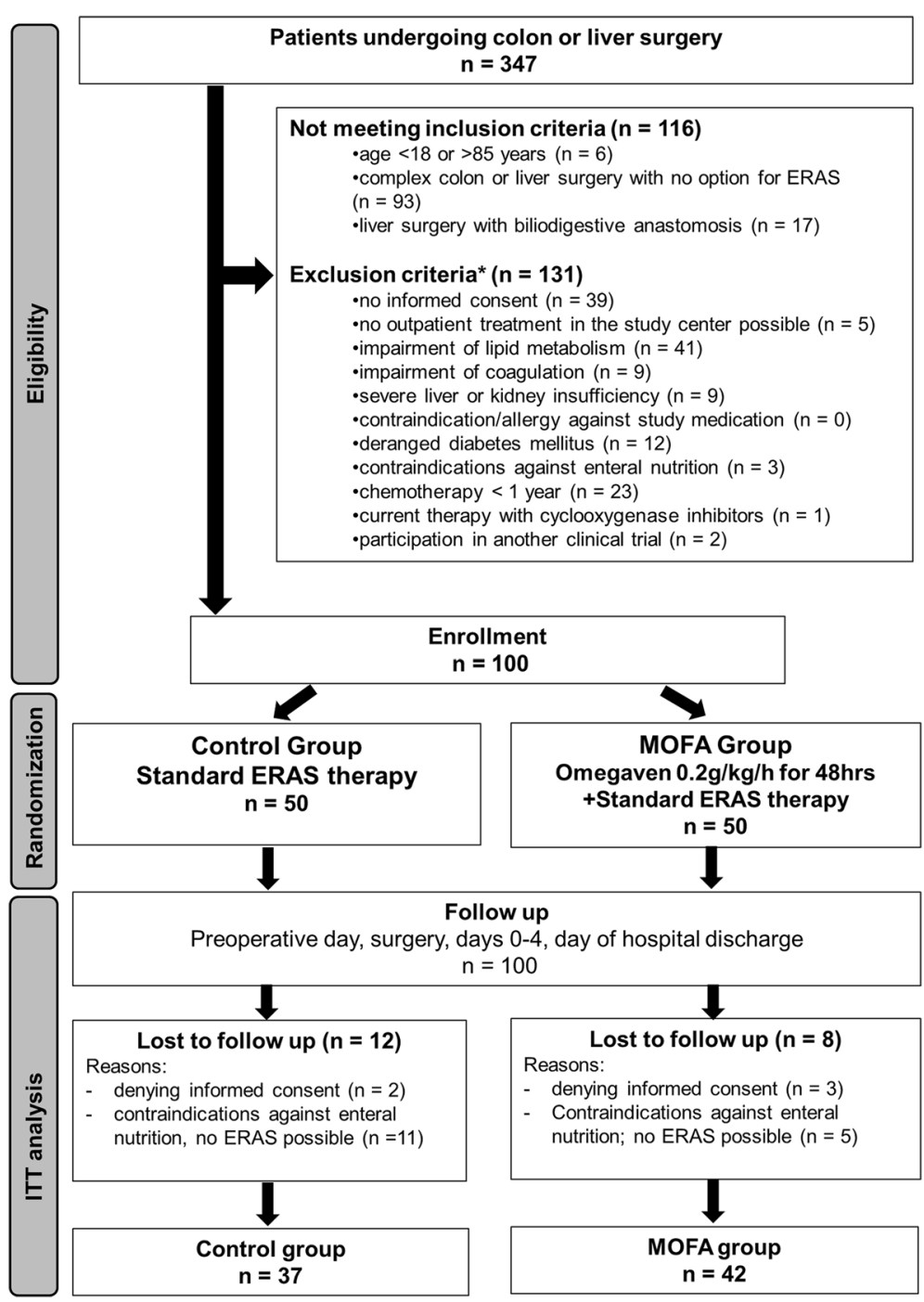

Fig. 2 Flowchart. Consecutive patients undergoing open liver or colon surgery were enrolled in the trial and randomized into ERAS and MOFA group. During the follow up period 28 patients were lost. Finally, 79 patients were analyzed in the per protocol analysis. ERAS: enhanced recovery after surgery concept, MOFA: metabolic optimized fast track concept, ITT: intention to treat

the first postoperative day by the treating intensivist although safety thresholds were not violated.

\section{Discussion}

The current trial is an independent investigator-initiated trial focusing on perioperative effects of the MOFA program. The trial was controlled and the interventions were simple and feasible, which improves external validity. The research question is highly relevant considering around 2.1 million patients treated for gastrointestinal diseases in Germany every year (according to hospital database of the German Federal Agency for Statistics Statistisches Bundesamt) resulting in approximately 8500 EUR up to 11,800 EUR costs per colon procedure and approximately 10,000 EUR up to 15,500 EUR costs per liver procedure [17-19].

\section{Major findings}

Major findings of the present trial are

1. The MOFA concept did not result in an improvement of intestine function represented as time to the first postoperative bowel movement in adult patients undergoing elective colon or hepatic surgery compared to standard ERAS therapy.

2. A faster recovery after surgery measured by the SAPS II score was not observed in the MOFA group compared to control group. 
Table 1 Baseline characteristics

\begin{tabular}{|c|c|c|}
\hline & Control group $(n=50)$ & MOFA group $(n=50)$ \\
\hline Age [years] & $61 \pm 12$ & $60 \pm 19$ \\
\hline \multicolumn{3}{|l|}{ Gender [no./\%] } \\
\hline Female & $16(32.0)$ & $18(36.0)$ \\
\hline Male & $34(68.0)$ & $32(64.0)$ \\
\hline Body height [m] & $1.73 \pm 0.09$ & $1.70 \pm 0.09$ \\
\hline Body weight [kg] & $82.7 \pm 16.2$ & $80.5 \pm 15.7$ \\
\hline BMI $\left[\mathrm{kg} / \mathrm{m}^{2}\right]$ & $27.6 \pm 4.7$ & $27.9 \pm 4.2$ \\
\hline \multicolumn{3}{|l|}{ Surgical procedure [no./\%] } \\
\hline Colon & $30(60.0)$ & $30(60.0)$ \\
\hline Liver & $20(40.0)$ & $20(40.0)$ \\
\hline \multicolumn{3}{|l|}{ Surgical technique [no./\%] } \\
\hline Laparoscopic & $6(12.0)$ & $5(10.0)$ \\
\hline Open & $43(86.0)$ & $45(90.0)$ \\
\hline Conversion $^{\mathrm{a}}$ & $1(2.0)$ & $0(0.0)$ \\
\hline \multicolumn{3}{|l|}{ ASA [no./\%] } \\
\hline । & $2(4.0)$ & $8(16.0)$ \\
\hline$\|$ & $29(58.0)$ & $30(60.0)$ \\
\hline III & $19(38.0)$ & $12(24.0)$ \\
\hline \multicolumn{3}{|l|}{ Preconditions } \\
\hline IDDM & $2(4.0)$ & $3(6.0)$ \\
\hline NIDDM & $6(12.0)$ & $4(8.0)$ \\
\hline Arterial hypertension & $29(58.0)$ & $25(50.0)$ \\
\hline Coronary heart disease & $2(4.0)$ & $6(12.0)$ \\
\hline COPD & $1(2.0)$ & $0(0.0)$ \\
\hline
\end{tabular}

Values are given as absolute number (percentage) or mean \pm standard deviation, as appropriate. ASA American Society of Anesthesiology physical status, BMI Body mass index, COPD Chronic obstructive pulmonary disease, IDDM Insulin dependent diabetes mellitus, MOFA Metabolic optimized fast track concept, NIDDM Non-insulin dependent diabetes mellitus, ${ }^{a}$ : intraoperative conversion from laparoscopic to open abdominal surgery
3. Omegaven ${ }^{\bullet}$ did not cause statistic significant impairment of coagulation or higher intraoperative blood loss compared to standard ERAS therapy.

\section{ERAS programs in abdominal surgery}

Careful analgesia, early enteral feeding and early mobilization are one of the key stones of modern fast track surgery [20]. The ERAS programs in abdominal surgery improve patient outcome and reduces costs [21, 22]. However, the comparability of the different ERAS approaches were questioned [22]. In the present trial our standard ERAS program consists of various interventions all recommended by the ERAS Society [20]. One essential part of our standard ERAS program is epidural anesthesia. Thoracic epidural anesthesia enhances gastrointestinal transit and reduces postoperative pain compared to conventional opioid analgesia [23]. The placement of epidural catheters in patients undergoing laparoscopic surgery might be obsolete considering risks and benefits as well as the alternative treatment with systemic opioids and/or local wound infiltration [24]. Furthermore, epidural anesthesia may be contraindicated in case of anticoagulant therapy [25], whereby various patients may be excluded from our proposed standard ERAS or MOFA program in case of early begin of anticoagulant therapy especially after laparoscopic surgery. In addition, new regional anesthetic techniques like the transverse abdominis plane block (TAP) or the paravertebral block, which are less invasive and have in case of TAP block less contraindications regarding anticoagulation showed comparable or better results with respect to analgesic effect or complications compared to TEA [26, 27]. However, a careful analgesia without influencing gastrointestinal transit may be a key concept independent of the type of analgesia. In addition, perioperative blood glucose control is essential. Kotagal and colleagues found in a retrospective cohort study in more than 40 , 000 patients undergoing abdominal surgery that perioperative hyperglycemia is associated with more adverse events even in patients without diabetes mellitus [28].

Table 2 Postoperative intestinal function

\begin{tabular}{llll}
\hline & Control group & MOFA group & $P$ value \\
\hline First bowel movement [h] & $74.5 \pm 30.4[38]$ & $66.4 \pm 29.2[43]$ & 0.163 \\
First discharge of gas [h] & $46.6 \pm 25.7[36]$ & $32.1 \pm 17.9[43]$ & $34.4 \pm 14.1[44]$ \\
Bowel sounds [h] & $40.8 \pm 14.6[40]$ & $0.0(0.0,0.0,1.0,3.0)[43]$ & 0.030 \\
Onset of enteral nutrition [days] & $0.0(0.0,0.0,1.0,5.0)[42]$ & $2.0(0.0,0.0,3.0,5.0)[43]$ & 0.042 \\
Bowel stimulation $^{\mathrm{a}}$ [days of onset] & $2.0(0.0,0.0,3.0,6.0)[39]$ & $22(51.2)[43]$ & 0.703 \\
Bowel stimulation $^{\mathrm{a}}$ [n] & $20(51.3)[39]$ & 1.000 &
\end{tabular}

Values are given as mean \pm standard deviation, median (minimum, 25\% percentile, $75 \%$ percentile, maximum) or absolute number (percentage) as appropriate. Number of analyzed patients can be found in brackets. Statistical significance was considered to be at two-sided $p<0.05$. Differences between groups were tested using log rank test and cox regression. Frequencies were analyzed using Fisher's Exact test or Chi square test, as appropriate. MOFA Metabolic optimized fast track concept, ${ }^{a}$ : bowel stimulation includes pharmacological and osmotic/mechanical stimulation 
TISS 28

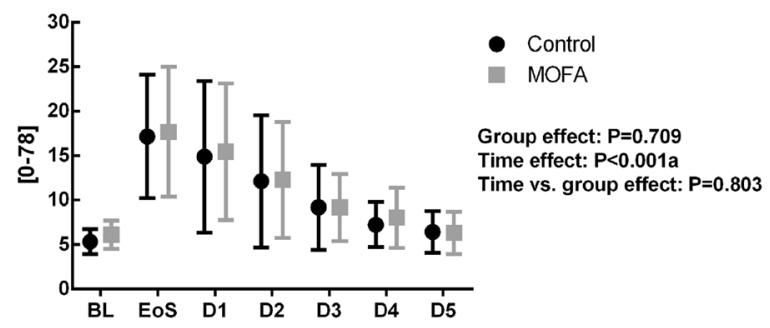

SAPS II

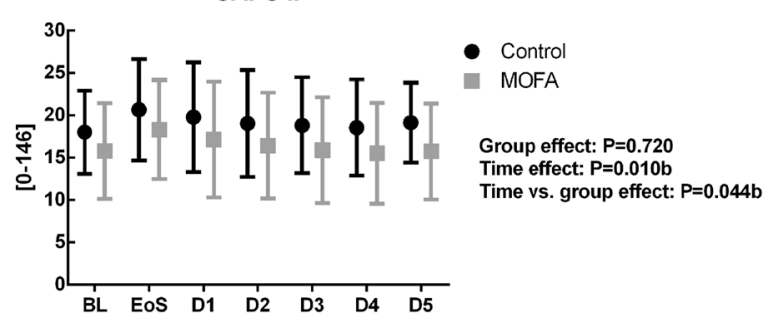

Fig. 3 TISS 28 and SAPS II score. Values are given as mean \pm standard deviation. Statistical significance was considered to be at two-sided $p<$ 0.05. Differences between groups, as well as time and time vs. group effect were tested using a general linear model with the respective baseline value as covariate. a: linear effect, b: quadratic effect, BL: baseline, EoS: Evening of surgery, D1-D5:postoperative day 1-5, respectively. SAPS: Simplified Acute Physiology Score, TISS: Simplified Therapeutic Scoring System, MOFA: metabolic optimized fast track concept

Therefore, the MOFA group received more insulin than the control group, but without resulting in lower blood glucose levels compared to control group. However, the average blood sugar level was below $8 \mathrm{mmol} / \mathrm{L}$ for most of the measurement points in both groups.

\section{Influence of omega-3 fatty acids}

Host defense is one of the most complexly regulated systems within the mammalian organism. Multitudes of back coupling mechanisms are responsible for up-regulating the immune response and for subsequent shut down of hostile responses during recovery. Through several positive feedback loops early host defense can induce hyperinflammatory states. Counterbalancing antagonistic systems are induced with a time delay. The early phase $(0-72 \mathrm{~h})$ is characterized by the predominance of pro-inflammatory eicosanoids, cytokines (e.g. TNF- $\alpha$, IL-1, IL-6, IL-8) and the in parts overlapping later phase by the predominance of anti-inflammatory cytokines (IL-4, IL-10, IL-13 and transforming growth factor- $\beta$ ) $[29,30]$. This time course of cytokine production can differ in patients with recurrent septic episodes.

The addition of Omegaven $^{\circ}$ to the standard ERAS treatment was promising due to several of beneficial immunomodulatory effects described in the literature.
Omegaven $^{\circ}$ is a lipid parenteral formula which contains $10 \mathrm{~g}$ fish oil $/ 100 \mathrm{ml}$ which correspondingly has 1.25 $2.82 \mathrm{~g}$ eicosapentaenoic acid (EPA, 20:5 n-3) and 1.44$3.09 \mathrm{~g}$ docosahexaenoic acid (DHA, 22:6 n-3) [31]. The n-6:n-3 PUFA ratio in Omegaven is 1:6-8.

In the recent years a couple of meta analyses in the field of intravenous n3FA administration shed light on their efficacy in surgical patients [32-34] as well as in critical illness [32, 35, 36]. Significantly lower infection rates, ICU stay, and length of hospital stay have concordantly been identified in three recent meta-analyses in surgical patients [32, 37, 38]. In this regard the posttraumatic metabolism after gastrointestinal cancer surgery was improved [4], which may in part be explained by resolvin $E_{1}$ activity [39]. This complex regulation is conferred by reduced release of pro-inflammatory arachidonic acid-derivatives and platlet-activating factor, and, on the other hand, by the amplification of anti-inflammatory EPA-derivatives, which lower the formation of cytokines such as TNF- $\alpha$ and IL-1 [40], without inhibiting phagocytosis, burst activity, or bactericidal activity [41]. The observed modulation of inflammation by n3FA, thus, cannot unequivocally be assigned to the pro- or anti-inflammatory side. While early pro- inflammatory eicosanoids are down- regulated, later on, cellular host defense mechanisms are augmented.

Besides the physiological rationale and experience from small clinical trials, Omegaven ${ }^{\circ}$ failed to improve postoperative intestinal function, inflammation and recovery from surgery in the present trial. We noticed neither an aggravation nor depression of immune function represented by cytokine levels and clinical relevant inflammatory parameters. In our opinion, four different reasons could explain those findings: 1) the trial was underpowered; 2) the dosage of Omegaven ${ }^{\circ}$ was too low; 3) the start of Omegaven was too late or the duration of administration was too short and 4) there is no clinical favourable effect of Omegaven ${ }^{\bullet}$ regarding intestinal function, inflammation or recovery after surgery.

Several factors may contribute to low power in the present trial. The estimated compensation of $6 \%$ for dropouts by three subjects per group was too low. Both the ERAS and MOFA program consists of various interventions, which may lead to patient inconvenience and therefore denying consent during the trial. In addition, the time of first bowel movement after colon surgery, which was used for sample size calculation may be different between from those after receiving liver surgery. However, since a statistic significant difference in the onset of flatulence was observed in the MOFA group, the authors consider the statistical power as main reason for failing the primary endpoint.

Finding the correct dosage of study medication in clinical trials is challenging. Heller et al. conducted a large 
Table 3 Duration of Surgery, Anesthesia, ICU and hospital stay, Complications of surgery

\begin{tabular}{|c|c|c|c|}
\hline & Control group & MOFA group & $P$ value \\
\hline \multicolumn{4}{|l|}{ Duration of surgery } \\
\hline \multicolumn{4}{|l|}{$[\mathrm{min}]$} \\
\hline All & $190(92,157,265,529)[50]$ & $215(89,158,310,560)[50]$ & 0.425 \\
\hline Colon & $175(92,150,203,293)[30]$ & $206(89,153,268,482)[30]$ & 0.240 \\
\hline Liver & $235(130,169,397,528)[20]$ & $221(92,166,336,560)[20]$ & 0.654 \\
\hline \multicolumn{4}{|c|}{ Duration of anesthesia } \\
\hline \multicolumn{4}{|l|}{ [min] } \\
\hline All & $276(180,239,355,620)[50]$ & $300(176,245,418,632)[50]$ & 0.491 \\
\hline Colon & $262(180,238,309,390)[30]$ & $289(187,243,361,570)[30]$ & 0.578 \\
\hline Liver & $335(196,237,537,620)[20]$ & $323(176,245,465,632)[20]$ & 0.186 \\
\hline \multicolumn{4}{|c|}{ Intraoperative blood loss } \\
\hline \multicolumn{4}{|l|}{$[\mathrm{ml}]$} \\
\hline All & $175(0,30,1025,3000)[50]$ & $400(0,30,950,3000)[49]$ & 0.775 \\
\hline Colon & $50(0,30,200,1150)[30]$ & $30(0,20,100,3000)[29]$ & 0.399 \\
\hline Liver & $1100(100,613,1438,3000)[20]$ & $950(50,525,1950,2820)[20]$ & 0.909 \\
\hline \multicolumn{4}{|c|}{ Need for transfusion ${ }^{\mathrm{b}}[\mathrm{no}]$} \\
\hline All & $10(20.0)[50]$ & $6(12.0)[50]$ & 0.414 \\
\hline Colon & $2(6.7)[30]$ & $1(3.3)[30]$ & 1.000 \\
\hline Liver & $8(40.0)[20]$ & $5(25.0)[20]$ & 0.501 \\
\hline \multicolumn{4}{|l|}{ Transfusion products } \\
\hline PRBC [units] & $4.0(2.0,2.5,5.0,8.0)[9]$ & $2.5(1.0,2.0,4.0,6.0)[8]$ & 0.288 \\
\hline FFP [units] & $4.0(2.0,2.0,5.25,9.0)[6]$ & $3.0(2.0,2.0,4.0,4.0)[4]$ & n.a. \\
\hline \multicolumn{4}{|c|}{ Cumulative fluid therapy } \\
\hline \multicolumn{4}{|l|}{ Crystalloids [ml] } \\
\hline All & $2500(500,1375,3500,5900)[50]$ & $1750(0,1100,2500,6500)[49]$ & 0.054 \\
\hline Colon & $1500(500,1000,2500,5500)[30]$ & $1500(0,1000,2250,6500)[29]$ & 0.758 \\
\hline Liver & $3500(2000,3000,4000,5900)[20]$ & $2263(0,1500,3500,5300)[20]$ & 0.004 \\
\hline \multicolumn{4}{|l|}{ Colloids [ml] } \\
\hline All & $500(0,500,1500,2500)[50]$ & $1000(0,500,1500,3000)[49]$ & 0.043 \\
\hline Colon & $500(0,0,1000,2000)[30]$ & $500(0,500,1000,3000)[29]$ & 0.005 \\
\hline Liver & $1500(500,500,1500,2500)[20]$ & $1500(0,500,2000,3000)[20]$ & 0.504 \\
\hline \multicolumn{4}{|c|}{ Hospital length of stay [days] } \\
\hline All & $8.0(6.0,8.0,9.0,20.0)[37]$ & $8.0(6.0,8.0,9.0,20.0)[43]$ & 0.466 \\
\hline Colon & $8.0(6.0,7.0,10.0,30.0)[23]$ & $8.0(6.0,7.0,9.0,12.0)[27]$ & 0.651 \\
\hline Liver & $8.0(6.0,8.0,9.0,20.0)[14]$ & $9.0(7.0,8.8,10.3,13.0)[16]$ & 0.860 \\
\hline \multicolumn{4}{|c|}{ ICU length of stay [hours] } \\
\hline All & $34(21,23,49,67)[14]$ & $27(12,20,45,80)[15]$ & 0.237 \\
\hline Colon & $49[1]$ & $30(12,13,43,44)[4]$ & n.a. \\
\hline Liver & $25(21,22,49,67)[13]$ & $27(18,21,45,88)[11]$ & 0.558 \\
\hline \multicolumn{4}{|c|}{ Postoperative surgical complications ${ }^{a}$ [no.] } \\
\hline Bleeding & $0(0.0)[50]$ & $1(2.0)[50]$ & 1.000 \\
\hline Insufficiency of & $4(8.0)[50]$ & $0(0.0)[50]$ & 0.118 \\
\hline \multicolumn{4}{|l|}{ anastomosis } \\
\hline Wound infection & $3(6.0)[50]$ & $0(0.0)[50]$ & 0.242 \\
\hline
\end{tabular}


Table 3 Duration of Surgery, Anesthesia, ICU and hospital stay, Complications of surgery (Continued)

\begin{tabular}{llll}
\hline & Control group & MOFA group & $P$ value \\
\hline Non-infective wound & $1(2.0)[50]$ & $1(2.0)[50]$ & \\
$\begin{array}{l}\text { complication } \\
\text { Biliary fistula }\end{array}$ & $0(0.0)[50]$ & $0(0.0)[50]$ & n.a. \\
Reoperation & $4(8.0)[50]$ & 0 (0.0) [50] & 0.118 \\
other & $2(4.0)[50]$ & $2(4.0)[50]$ & 1.000 \\
In-hospital death [no.] & $1(2.0)[50]$ & $0(0.0)[50]$ & 1.000 \\
\hline
\end{tabular}

Values are given as mean \pm standard deviation, median (minimum, $25 \%$ percentile, $75 \%$ percentile, maximum) or absolute number (percentage) as appropriate. Number of analyzed patients can be found in brackets. Statistical significance was considered to be at two-sided $p<0.05$. Differences between groups were tested using a Student's t-test or Mann-Whitney $U$ test as appropriate. Frequencies were analyzed using Chi-square or Fisher's Exact test, as appropriate. FFP Fresh frozen plasma, ICU Intensive care unit, MOFA Metabolic optimized fast track concept, PRBC packed red blood cells. ${ }^{\mathrm{a}}$ : Postoperative surgical complications are provided during whole hospital stay or until dropout. ${ }^{\text {b }}$ : need for transfusion is given during whole hospital stay or until dropout

observational study of intravenous fish oils in 661 patients from 82 German hospitals [12]. Doses between $0.05-0.2 \mathrm{~g} / \mathrm{kg} / \mathrm{day}$ of fish oil were given to mixed hospital patients for at least 3 days. An average of $0.11 \mathrm{~g} / \mathrm{kg} /$ day [range $0.005-0.426 \mathrm{~g} / \mathrm{kg} / \mathrm{day}$ ] was administered for an average of $8.7 \pm 7.5$ days. Fish oil administration significantly lowered hospital mortality from 18.9 to $12 \%$ as predicted by SAPS II scores. Fish oil doses $>0.05 \mathrm{~g} / \mathrm{kg} /$ day significantly decreased length of ICU and hospital stay. Doses between $0.1-0.2 \mathrm{~g} / \mathrm{kg} /$ day was associated with significantly higher survival rates compared to doses $<0.05 \mathrm{~g} / \mathrm{kg} /$ day. Finally, a lowered antibiotic demand of $26 \%$ was observed in patients receiving 0.15 $0.2 \mathrm{~g} / \mathrm{kg} /$ day of fish oil. Based on this trial we opted for Omegaven $^{\circ}$ dosage. Wang et al. administered up to $10 \mathrm{~g} /$ day by giving $0.15-0.2 \mathrm{~g} / \mathrm{kg} /$ day of fish oil from Omegaven $^{\circ}$ in 40 adult patients with severe acute pancreatitis [42]. Clinical parameters were measured on day 6 , the following day after the intervention. In the fish oil treated group, EPA was significantly higher in plasma membranes, CRP concentrations were lower and oxygenation index were higher compared to control. However, an optimal dose finding trial should be performed before conducting future clinical trials involving intravenous Omegaven ${ }^{\circ}$.

The optimal time point for beginning, the duration as well as the route of administration of n3FA is still crucial. As shown by Brower et al., the intestinal resorption of n3FA is not sufficient in all patients during systemic inflammation [43]. Therefore, an intravenous administration is favorable. The optimal start and duration of supplementation with n3FA was chosen to cover the peak of inflammatory response. However, an orally supplementation in a longer period before surgery may enhance beneficial effects of n3FA. Furthermore, recent orally available n3FA formulas consisting of monoglycerids attached with EPA (EPA/DHA ratio 10:1) and less fishy taste may be opted for oral supplementation before and after surgery [44].
Recent randomized controlled trials showed no effect on mortality in critical ill or cancer patients undergoing elective surgery $[45,46]$. These findings are supported by metaanalysis, which showed no benefit on mortality or infectious complications [36]. However, there is the possibility that n3FA do not have an effect on intestinal function, resolution of inflammation or recovery after surgery in the currently investigated patient collective. Nevertheless, in awareness that the present trial is underpowered a generalization of the results cannot be made.

\section{Safety of Omegaven ${ }^{\oplus}$}

The anti-thrombotic and anti-atherogenic effects of fish oil have raised potential safety concerns due to decreased blood viscosity, which could decrease blood clotting [47]. To date, negative clinical data is sparse for prolonged bleeding times for fish oil alone, or in combination with blood thinners [48-50] with the exception of some isolated case reports on possible interactions between warfarin and/or trazodone and/ or aspirin with oral fish oil [51-54]. Fish oil supplementation has shown to be safe in various RCTs in conjunction with and without anti-coagulant therapy. In the GISSI multi-center trial, no negative blood thinning interactions were reported in over 10,000 post-myocardial patients that consumed around $1 \mathrm{~g} /$ day DHA + EPA from fish oil in combination with blood thinning agents for 3-5 years [55]. In high risk surgical patients undergoing major vascular surgeries, femoral oral artery puncture or percutaneous transluminar coronary angioplasty, Harris reported on 20 studies which included over 4300 subjects that supplemented between 1 and $21 \mathrm{~g} /$ day fish oil concomitantly with blood-thinning medications in subjects [48]. Of these, 5 bleeding complications were reported and no clinically relevant extended bleeding times were documented.

Statistical significant undesirable effects of n3FA on coagulation or bleeding disorders did not occur in the 
present trial. However, because the present trial is underpowered, further trials are needed to investigate the safety of Omegaven ${ }^{\circ}$ regarding impairment of coagulation.

\section{Limitations}

The present trial has several limitations. First, the trial was an open label trial, which may have and severe impact on outcome [56]. Blinding of outcome assessors and patients via a double dummy design with sham infusion of crystalloid infusion would have improved drastically trial costs for camouflaging study medication. Second, the trial is underpowered due to the high rate of dropouts in both groups as discussed before. Third, liver and colon surgery may be too heterogeneous since higher blood loss and transfusion are more likely to occur in liver surgery. We opted for a stratified randomization strategy, which enrolled an equal number of patients with liver resection in both groups which enhanced comparability and improved external validity of the trial. Forth, only a few patients received laparoscopic surgery in our center, whereby the use of laparoscopic techniques is improving for simple and also complex liver and colon surgery. In addition, less surgical trauma may be caused by laparoscopic techniques resulting in moderated inflammatory response confounding the study results. Fifth, the placement of epidural anesthesia in patients undergoing laparoscopic surgery might be obsolete considering risks and benefits as well as the alternative treatment with systemic opioids and/or local wound infiltration as mentioned before [24]. Furthermore, epidural anesthesia may be contraindicated in case of anticoagulant therapy [25], whereby various patients may be excluded from our proposed standard ERAS or MOFA program in case of early begin of anticoagulant therapy especially after laparoscopic surgery. However, considering a trial design without obligate TEA, an imbalance between groups with different number of TEA would be a clear confounder, due to enhanced bowel movement and shorter hospital stay by TEA after abdominal surgery [44]. Sixth, the evaluation of safety thresholds might be difficult in case of large blood loss caused by surgery.

\section{Implications for further studies}

Further trials investigating the influence of n3FA in a larger patient collective undergoing abdominal surgery are warranted. Such a trial should be randomized controlled with a double dummy design, focusing on the effects of omega- 3 fatty acids in patients undergoing open abdominal surgery. An optimal dose finding trial as well as the optimal time point for beginning of the Omegaven ${ }^{\circ}$ treatment is necessary. A preoperative start of orally n3FA supplementation for instance two weeks before surgery and a longer supplementation period of for instance 4 weeks postoperatively may enhance the immunomodulatory effect of n3FA. An increase in daily dosage to $0.5 \mathrm{~g} / \mathrm{kg} / \mathrm{d}$ fish oil may result in a clinical relevant improvement of intestinal function and faster recovery after surgery.

\section{Conclusions}

The metabolic optimized fast track concept did not result in an improvement of intestine function or faster recovery after elective colon or liver surgery compared to standard enhanced recovery after surgery program. Omega- 3 fatty acids showed no impairment of coagulation or improved resolution of inflammation. Further trials in a larger patient collective are needed to investigate potential beneficial effects of omega- 3 fatty acids in abdominal surgery.

\section{Additional file}

Additional file 1: Supplemental digital content for this manuscript is provided. This file contains a detailed description of methods (Table S1. Exclusion criteria, Table S2. Detailed description of study intervention, Table S3. Blood glucose management) as well as additional results (Figure S1. Blood glucose levels, Figure S2. Coagulation parameters, Figure S3: Parameters of liver injury and function, Figure S4. Inflammation, Table S4. Hemodynamic data, Table S5. Insulin therapy, Table S6. Additional clinical chemistry and blood count). (DOCX $452 \mathrm{~kb}$ )

\section{Abbreviations \\ aPTT: Activated partial thromboplastin time; BfArM: Bundesinstitut für Arzneimittelsicherheit und Medizinprodukte; CONSORT: Consolidated Standards of Reporting Trials; CRP: C-reactive protein; DHA: Docosahexaenoic acid; EPA: Eicosapentaenoic acid; ERAS: Enhanced recovery after surgery; EuDraCT: European Union Drug Review Agency Clinical Trials; GC: Glucose control; ICU: Intensive care unit; IL: Interleukin; MOFA: Metabolic Optimized FAst track concept; n3FA: Omega-3 fatty acids; PCT: Procalcitonin; PT: Prothrombin time; RCT: Randomized controlled trial; SAPS: Simplified Acute Physiology Score; SOFA: Sequential/Sepsis related Organ Failure Assessment; TAP: Transverse abdominis plane block; TEA: Thoracic epidural anesthesia; TISS: Simplified Therapeutic Scoring System; TNF-a: Tumor necrosis factor-alpha}

\section{Acknowledgements}

The authors thank the anesthetic and surgical staff related to the Department of Visceral, Thoracic and Vascular Surgery, University Hospital Carl Gustav Carus, Technische Universität Dresden, Dresden, Germany. The authors express their special gratitude to the doctoral students of the Department of Anesthesiology and Critical Care Medicine, University Hospital Carl Gustav Carus, Technische Universität Dresden, Dresden, Germany; Katja Dubois, Sebastian Weidauer, Beatrice Schubert, and Heike Köhler, who helped with data collection.

\section{Authors' contributions}

$\mathrm{CU}$ prepared the manuscript and performed statistical analysis. TR, AD and SS collected data and contributed to the manuscript. TK helped with manuscript preparation. ARH designed the study protocol, collected data and contributed to manuscript preparation. All authors have read and approved the final version of the manuscript.

\section{Funding}

The present trial was supported by Fresenius Kabi Deutschland AG, Bad Homburg, Germany, who provided the trial medication, but had no part in study design, data collection, interpreting the results or preparing the manuscript. We acknowledge support by the Open Access Publication Funds of the SLUB/TU Dresden. 


\section{Availability of data and materials}

The datasets used and/or analyzed during the current study are available from the corresponding author on reasonable request.

\section{Ethics approval and consent to participate}

The present trial was approved by the Bundesinstitut für Arzneimittelsicherheit und Medizinprodukte (BfArM, GZ 61-3910-4031476), and the local Institutional Review Board "Ethikkommission an der Technischen Universität Dresden" (AZ 193112005). Written informed consent was obtained from all participants prior to trial enrollment.

\section{Consent for publication}

Not applicable.

\section{Competing interests}

ARH received study grants by Fresenius Kabi Deutschland AG, Bad Homburg, Germany and speaker honoraria by CSL- Behring, Marburg, Germany. TK received speaker honoraria from Fresenius Kabi Deutschland AG, Bad Homburg, Germany. CU, TR, AD, and SS declare that they have no competing interests.

\section{Author details}

${ }^{1}$ Department of Anaesthesiology and Critical Care Medicine, University Hospital Carl Gustav Carus at the Technische Universität Dresden, Fetscherstr. 74, 01307 Dresden, Germany. ${ }^{2}$ Department of Gastrointestinal, Thoracic and Vascular Surgery, University Hospital Carl Gustav Carus at the Technische Universität Dresden, Dresden, Germany. ${ }^{3}$ Department of General Surgery, University Hospital of Friedrich-Alexander-University, Erlangen, Germany. ${ }^{4}$ Department of Thorax, Vascular and Endovascular Surgery, Chemnitz Hospital, Chemnitz, Germany. ${ }^{5}$ Department of Anaesthesiology and Surgical Intensive Care Medicine, University Hospital Augsburg, Augsburg, Germany.

Received: 13 May 2019 Accepted: 6 August 2019

Published online: 17 August 2019

\section{References}

1. Ni TG, Yang HT, Zhang H, Meng HP, Li B. Enhanced recovery after surgery programs in patients undergoing hepatectomy: a meta-analysis. World J Gastroenterol. 2015;21(30):9209-16.

2. Kehlet $\mathrm{H}$. Fast-track surgery-an update on physiological care principles to enhance recovery. Langenbeck's Arch Surg. 2011;396(5):585-90.

3. Heller AR, Theilen HJ, Koch T. Fish or chips? News Physiol Sci. 2003;18:50-4.

4. Heller AR, Rossel T, Gottschlich B, Tiebel O, Menschikowski M, Litz RJ, Zimmermann T, Koch T. Omega-3 fatty acids improve liver and pancreas function in postoperative cancer patients. Int J Cancer. 2004;111(4):611-6.

5. Heller AR. Intravenous fish oil in adult intensive care unit patients. World Rev Nutr Diet. 2015:112:127-40.

6. Calder PC. Mechanisms of action of (n-3) fatty acids. J Nutr. 2012;142(3): 5925-9S.

7. Heller A, Koch T, Schmeck J, van Ackern K. Lipid mediators in inflammatory disorders. Drugs. 1998;55(4):487-96.

8. Serhan CN, Chiang N. Resolution phase lipid mediators of inflammation: agonists of resolution. Curr Opin Pharmacol. 2013;13(4):632-40.

9. Breil I, Koch T, Heller A, Schlotzer E, Grunert A, van Ackern K, Neuhof H. Alteration of $n-3$ fatty acid composition in lung tissue after short-term infusion of fish oil emulsion attenuates inflammatory vascular reaction. Crit Care Med. 1996;24(11):1893-902.

10. Koch TH, A Breil I, van Ackern K, Neuhof H. Alterations of pulmonary capillary filtration and leukotriene synthesis due to infusion of a lipid emulsion enriched with omega 3 fatty acids. Clin Intensive Care. 1995;6: 112-20.

11. Berger MM, Delodder F, Liaudet L, Tozzi P, Schlaepfer J, Chiolero RL, Tappy $\mathrm{L}$. Three short perioperative infusions of n-3 PUFAs reduce systemic inflammation induced by cardiopulmonary bypass surgery: a randomized controlled trial. Am J Clin Nutr. 2013;97(2):246-54.

12. Heller AR, Rossler S, Litz RJ, Stehr SN, Heller SC, Koch R, Koch T. Omega-3 fatty acids improve the diagnosis-related clinical outcome. Crit Care Med. 2006:34(4):972-9.

13. Pradelli L, Eandi M, Povero M, Mayer K, Muscaritoli M, Heller AR, FriesSchaffner E. Cost-effectiveness of omega-3 fatty acid supplements in parenteral nutrition therapy in hospitals: a discrete event simulation model. Clin Nutr. 2014;33(5):785-92.

14. Le Gall JR, Lemeshow S, Saulnier F. A new simplified acute physiology score (SAPS II) based on a European/north American multicenter study. JAMA. 1993;270(24):2957-63.

15. Miranda DR, de Rijk A, Schaufeli W. Simplified therapeutic intervention scoring system: the TISS-28 items--results from a multicenter study. Crit Care Med. 1996;24(1):64-73.

16. Schulz KF, Altman DG, Moher D, Group C. CONSORT 2010 statement: updated guidelines for reporting parallel group randomised trials. BMJ. 2010;340:c332

17. [G-DRG-Reportbrowser 2019] [https://www.g-drg.de/Datenbrowser_und_ Begleitforschung/G-DRG-Report-Browser/G-DRG-Report-Browser_2019].

18. [DRG Fallpauschalenkatalog 2019] [https://www.g-drg.de/G-DRG-System_2 019/Fallpauschalen-Katalog/Fallpauschalen-Katalog_2019].

19. [Krankenhausstatistik mit Fallzahlen/DRGs] [https://www.destatis.de/DE/ Methoden/Qualitaet/Qualitaetsberichte/Gesundheit/fallpauschalenbezognek rankenhausstatistk.html].

20. Gustafsson UO, Scott MJ, Hubner M, Nygren J, Demartines N, Francis N Rockall TA, Young-Fadok TM, Hill AG, Soop M, et al. Guidelines for perioperative Care in Elective Colorectal Surgery: enhanced recovery after surgery (ERAS((R))) society recommendations: 2018. World J Surg. 2019;43(3): 659-95.

21. Lemanu DP, Singh PP, Stowers MD, Hill AG. A systematic review to assess cost effectiveness of enhanced recovery after surgery programmes in colorectal surgery. Color Dis. 2014;16(5):338-46.

22. Greer NL, Gunnar WP, Dahm P, Lee AE, MacDonald R, Shaukat A, Sultan S, Wilt TJ. Enhanced recovery protocols for adults undergoing colorectal surgery: a systematic review and Meta-analysis. Dis Colon Rectum. 2018; 61(9):1108-18

23. Guay J, Nishimori M, Kopp S. Epidural local anaesthetics versus opioid-based analgesic regimens for postoperative gastrointestinal paralysis, vomiting and pain after abdominal surgery. Cochrane Database Syst Rev. 2016;7: CD001893.

24. Keller DS, Pedraza R, Tahilramani RN, Flores-Gonzalez JR, Ibarra S, Haas EM. Impact of long-acting local anesthesia on clinical and financial outcomes in laparoscopic colorectal surgery. Am J Surg. 2017;214(1):53-8.

25. Gogarten W, Vandermeulen E, Van Aken H, Kozek S, Llau JV, Samama CM. European Scoeity of a: regional anaesthesia and antithrombotic agents: recommendations of the European society of anaesthesiology. Eur J Anaesthesiol. 2010;27(12):999-1015.

26. El-Boghdadly K, Madjdpour C, Chin KJ. Thoracic paravertebral blocks in abdominal surgery - a systematic review of randomized controlled trials. $\mathrm{Br}$ J Anaesth. 2016;117(3):297-308

27. Baeriswyl M, Zeiter F, Piubellini D, Kirkham KR, Albrecht E. The analgesic efficacy of transverse abdominis plane block versus epidural analgesia: a systematic review with meta-analysis. Medicine (Baltimore). 2018;97(26):e11261

28. Kotagal M, Symons RG, Hirsch IB, Umpierrez GE, Dellinger EP, Farrokhi ET, Flum DR, Collaborative S-C. Perioperative hyperglycemia and risk of adverse events among patients with and without diabetes. Ann Surg. 2015;261(1): 97-103.

29. Fullerton JN, O'Brien AJ, Gilroy DW. Lipid mediators in immune dysfunction after severe inflammation. Trends Immunol. 2014;35(1):12-21.

30. Ayala A, Chung CS, Grutkoski PS, Song GY. Mechanisms of immune resolution. Crit Care Med. 2003;31(8 Suppl):S558-71.

31. Kabi F. Omegaven product monograph; 2009.

32. Pradelli L, Mayer K, Muscaritoli M, Heller AR. N-3 fatty acid-enriched parenteral nutrition regimens in elective surgical and ICU patients: a metaanalysis. Crit Care. 2012;16(5):R184.

33. Yu J, Liu L, Zhang Y, Wei J, Yang F. Effects of omega-3 fatty acids on patients undergoing surgery for gastrointestinal malignancy: a systematic review and meta-analysis. BMC Cancer. 2017;17(1):271.

34. Li NN, Zhou Y, Qin XP, Chen Y, He D, Feng JY, Wu XT. Does intravenous fish oil benefit patients post-surgery? A meta-analysis of randomised controlled trials. Clin Nutr. 2014;33(2):226-39.

35. Manzanares W, Dhaliwal R, Jurewitsch B, Stapleton RD, Jeejeebhoy KN, Heyland DK. Parenteral fish oil lipid emulsions in the critically ill: a systematic review and meta-analysis. JPEN J Parenter Enteral Nutr. 2014;38(1):20-8.

36. Palmer AJ, Ho CK, Ajibola O, Avenell A. The role of omega-3 fatty acid supplemented parenteral nutrition in critical illness in adults: a systematic review and meta-analysis. Crit Care Med. 2013;41(1):307-16. 
37. Chen W, Jiang H, Zhou ZY, Tao YX, Cai B, Liu J, Yang H, Lu CD, Zeng J. Is omega-3 fatty acids enriched nutrition support safe for critical ill patients? A systematic review and meta-analysis. Nutrients. 2014;6(6):2148-64.

38. Manzanares W, Dhaliwal R, Jurewitsch B, Stapleton RD, Jeejeebhoy KN, Heyland DK. Alternative lipid emulsions in the critically ill: a systematic review of the evidence. Intensive Care Med. 2013:39(10):1683-94.

39. Arita M, Bianchini F, Aliberti J, Sher A, Chiang N, Hong S, Yang R, Petasis NA, Serhan CN. Stereochemical assignment, antiinflammatory properties, and receptor for the omega-3 lipid mediator resolvin E1. J Exp Med. 2005;201(5): 713-22.

40. Molvig J, Pociot F, Worsaae H, Wogensen LD, Baek L, Christensen P, Mandrup-Poulsen T, Andersen K, Madsen P, Dyerberg J, et al. Dietary supplementation with omega-3-polyunsaturated fatty acids decreases mononuclear cell proliferation and interleukin-1 beta content but not monokine secretion in healthy and insulin-dependent diabetic individuals. Scand J Immunol. 1991;34(4):399-410.

41. Palombo JD, DeMichele SJ, Boyce PJ, Lydon EE, Liu JW, Huang YS, Forse RA, Mizgerd JP, Bistrian BR. Effect of short-term enteral feeding with eicosapentaenoic and gamma-linolenic acids on alveolar macrophage eicosanoid synthesis and bactericidal function in rats. Crit Care Med. 1999; 27(9):1908-15.

42. Wang X, Li W, Li N, Li J. Omega-3 fatty acids-supplemented parenteral nutrition decreases hyperinflammatory response and attenuates systemic disease sequelae in severe acute pancreatitis: a randomized and controlled study. JPEN J Parenter Enteral Nutr. 2008;32(3):236-41.

43. Bower RH, Cerra FB, Bershadsky B, Licari JJ, Hoyt DB, Jensen GL, Van Buren $C T$, Rothkopf MM, Daly JM, Adelsberg BR. Early enteral administration of a formula (impact) supplemented with arginine, nucleotides, and fish oil in intensive care unit patients: results of a multicenter, prospective, randomized, clinical trial. Crit Care Med. 1995;23(3):436-49.

44. Guertin MH, Robitaille K, Pelletier JF, Duchesne T, Julien P, Savard J, Bairati I, Fradet $\mathrm{V}$. Effects of concentrated long-chain omega-3 polyunsaturated fatty acid supplementation before radical prostatectomy on prostate cancer proliferation, inflammation, and quality of life: study protocol for a phase IIb, randomized, double-blind, placebo-controlled trial. BMC Cancer. 2018;18(1):64.

45. Hanai N, Terada H, Hirakawa H, Suzuki H, Nishikawa D, Beppu S, Hasegawa Y. Prospective randomized investigation implementing immunonutritional therapy using a nutritional supplement with a high blend ratio of omega-3 fatty acids during the perioperative period for head and neck carcinomas. Jpn J Clin Oncol. 2018;48(4):356-61.

46. Kagan I, Cohen J, Stein M, Bendavid I, Pinsker D, Silva V, Theilla M, Anbar R, Lev S, Grinev M, et al. Preemptive enteral nutrition enriched with eicosapentaenoic acid, gamma-linolenic acid and antioxidants in severe multiple trauma: a prospective, randomized, double-blind study. Intensive Care Med. 2015;41(3):460-9.

47. Eritsland J, Arnesen H, Seljeflot I, Hostmark AT. Long-term metabolic effects of n-3 polyunsaturated fatty acids in patients with coronary artery disease. Am J Clin Nutr. 1995;61(4):831-6.

48. Harris WS. Expert opinion: omega-3 fatty acids and bleeding-cause for concern? Am J Cardiol. 2007;99(6A):44C-6C.

49. Bays HE. Safety considerations with omega-3 fatty acid therapy. Am J Cardiol. 2007:99(6A):35C-43C.

50. Hooper L, Thompson RL, Harrison RA, Summerbell CD, Moore $H_{\text {, }}$ Worthington HV, Durrington PN, Ness AR, Capps NE, Davey Smith G, et al. Omega 3 fatty acids for prevention and treatment of cardiovascular disease. Cochrane Database Syst Rev. 2004;(4):CD003177.

51. Buckley MS, Goff AD, Knapp WE. Fish oil interaction with warfarin. Ann Pharmacother. 2004;38(1):50-2.

52. Haller C, Kearney T, Bent S, Ko R, Benowitz N, Olson K. Dietary supplement adverse events: report of a one-year poison center surveillance project. J Med Toxicol. 2008;4(2):84-92.

53. Jalili M, Dehpour AR. Extremely prolonged INR associated with warfarin in combination with both trazodone and omega-3 fatty acids. Arch Med Res. 2007;38(8):901-4.

54. McClaskey EM, Michalets EL. Subdural hematoma after a fall in an elderly patient taking high-dose omega-3 fatty acids with warfarin and aspirin: case report and review of the literature. Pharmacotherapy. 2007;27(1):152-60.

55. Gruppo Italiano per lo Studio della Sopravvivenza nell'Infarto miocardico. Dietary supplementation with n-3 polyunsaturated fatty acids and vitamin E after myocardial infarction: results of the GISSI-Prevenzione trial. Lancet. 1999;354(9177):447-55.
56. Higgins JPT, Green S. Cochrane Handbook for Systematic Reviews of Interventions Version 5.1.0 [updated March 2011]; 2011.

\section{Publisher's Note}

Springer Nature remains neutral with regard to jurisdictional claims in published maps and institutional affiliations.

\section{Ready to submit your research? Choose BMC and benefit from:}

- fast, convenient online submission

- thorough peer review by experienced researchers in your field

- rapid publication on acceptance

- support for research data, including large and complex data types

- gold Open Access which fosters wider collaboration and increased citations

- maximum visibility for your research: over $100 \mathrm{M}$ website views per year

At BMC, research is always in progress.

Learn more biomedcentral.com/submissions 